\title{
Significance of cutting plane in liquid metal embrittlement severity quantification
}

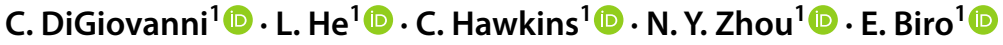

Received: 12 September 2020 / Accepted: 26 April 2021

Published online: 10 May 2021

(c) The Author(s) 2021

OPEN

\begin{abstract}
The automotive industry is turning to advanced high strength steels (AHSS) to reduce vehicle weight and increase fuel efficiency. However, the zinc coating on AHSS can cause liquid metal embrittlement (LME) cracking during resistance spot welding. To understand the problem, the severity of the cracking must be measured. Typically, this is done from the weld cross-section. Currently, there is no standard procedure to determine which plane through the weld must be examined to gauge cracking severity, leading to a variety of practices for choosing a cutting plane. This work compares the magnitude and variability of LME severity measured from the plane of exhibiting the most severe surface cracking to arbitrarily chosen planes. The plane exhibiting the most severe cracks had more and longer cracks on the cross-section than the arbitrarily chosen plane, resulting in a higher crack severity measurement. This higher absolute measurement increased the relative accuracy of the examination, allowing for fewer welds to be examined to precisely determine the effect of LME mitigation methods on cracking severity, how welding parameters affect LME cracking severity and the predicted LME affected strength of a particular weld.
\end{abstract}

Keywords Resistance spot welding (RSW) · Liquid metal embrittlement (LME) · Metallographic preparation · Quantitative metallography

\section{Introduction}

Advanced high strength steel (AHSS) is being adopted to increase material strength for automotive parts. However, when some of these steels are resistance spot welded (RSW) during automotive assembly, the protective zinc coating on the steel surface melts and enters the grain boundaries of the steel substrate, leading to intergranular cracking [1-3]. This is known as liquid metal embrittlement (LME), and may decrease spot weld strength [4-6].

Due to concerns about the impact of LME cracks on RSW strength, automotive standards are strict on how large surface cracks may be before welds are no longer fitfor-service [7]. It should also be noted that no cracks in the weld shoulder are permissible, which is where LME cracks are often seen are seen [3]. These standards are in-line with research that shows that the depth of shoulder cracks can affect the degree of strength loss exhibited by welds with LME cracks when compared to uncracked welds $[8,9]$. Furthermore, these studies showed LME shoulder cracks less than 300-325 $\mu \mathrm{m}$ (for these specific joints) did not affect joint strength.

Precisely correlating crack depth to weld strength degradation for a particular weld is unrealistic because there is large weld-to-weld variation in LME crack severity and location [10], and in a manufacturing setting, there are insufficient resources to measure crack depths from all welds. However, considering that a particular combination of welding

E. Biro, ejbiro@uwaterloo.ca; C. DiGiovanni, ctdigiov@uwaterloo.ca; L. He, I39he@uwaterloo.ca; C. Hawkins, cphawkins@uwaterloo.ca; N. Y. Zhou, nzhou@uwaterloo.ca| ${ }^{1}$ Department of Mechanical and Mechatronics Engineering, University of Waterloo, Waterloo, ON, Canada. 
parameters will result in a repeatable depth/quantity distribution of LME cracks in multiple welds $[6,11]$, cracking severity may be classified based on the number and depth of the entire population of cracks found in a particular weld. This approach was used by Wintjes et al. [6] to correlate an LME cracking severity index to welds strength degradation.

With the understanding of how LME crack dimensions and quantity can affect weld strength, as well as how LME cracks may be characterized in a repeatable way, weld crack measurements may be used to optimize the welding process by minimize cracking, and gain further understanding of how crack orientation and loading configuration affect joint strength [9]. However, to do this, a method to measure cracking severity must be determined. The only way to measure the entire population of cracks within a weld is by using volumetric techniques such as $\mathrm{x}$-ray computer tomography $(C T)$ scanning $[12,13]$, or using serial metallography where the specimens are repeatedly ground a finite amount and successive planes are inspected for cracks [14]. However, these techniques are very time intensive and are not well suited to characterizing LME due number of replicates needed to accurately measure crack distribution because of high weld-to-weld variability. Excluding volumetric cracking measurement means that planar measurement is the only viable option to characterize cracking distribution. Many studies have assessed cracking susceptibility from plan view (as seen from the top of the weld) using methods such as optical microscopy $[1,15,16]$, liquid dye penetrant $[2,17]$, radiography [18], fluorescent magnetic particle detection [19] and pulsed eddy current thermography [20]. However, using these techniques only views the weld surface. No information about crack depth may be learned, which gives no insight into whether the observed LME cracks will affect weld strength. Therefore, to balance testing efficiency and insight into post-welding properties, LME cracks must be measured from the weld cross-section.

For the reasons stated above, many studies have measured LME cracking severity from the weld cross-section. However, in the literature there seems to be two major philosophies on choosing the cross-section plane; these are the plane intersecting the most severe cracks $[1,6,19,21-23]$, and a plane with a fixed orientation to the welding coupon $[16,24,25]$. In the above cases the cross-section plane intersected the center of the weld (lying along the weld diameter). Although, it should be noted that in many cases, the method used to determine the cross-section plane was not described $[2,26,27]$, and in one case, the cross-section plane did not necessarily bisect the weld [28]. Considering the importance that LME crack dimensions has on weld properties, it is important to understand how measurements taken from a particular cross-section plane are representative of the crack distribution for a set of welds made with a single set of welding parameters. Furthermore, considering the large amount of research in this area, it must be known if results from various studies may be compared. This study examines the distribution of LME cracks in a set of welds made with standard test of welding conditions as measured from both cross-sections planes intersecting the most severe surface crack and those that are chosen randomly. The cracking severity from both measurements are compared in terms of crack depth and frequency, and recommendations are put forward as how weld cross-sections should be prepared for analysis to ensure the most representative results while minimizing testing effort.

\section{Materials and methods}

A transformation induced plasticity steel with a tensile strength of $1100 \mathrm{MPa}$ (TRIP1100) and thickness of 1.6 $\mathrm{mm}$ was used in this study. This material is susceptible to LME cracking and is of interest to industry. The chemical composition of the steel may be found in Table 1. Twelve samples were welded using a DC pedestal spot welder with an air over oil force actuation system. All welds were made at the expulsion current, with the remaining welding parameters were selected from AWS D8.9 [29] and are listed in Table 2.

After welding, six samples were randomly chosen and cross-sectioned along arbitrary cutting planes through the center of the weld nugget. This method of plane selection is called the arbitrary cutting plane method. The remaining six samples were first inspected for surface cracks using a stereomicroscope, then the cutting planes were chosen so that they would intersect through both the center of the weld nugget and the greatest number of surface cracks, as shown in Fig. 1. This is referred to as the selected cutting plane method.

Table 2 Welding parameters in accordance with AWS D8.9 [29]

\begin{tabular}{llllll}
\hline $\begin{array}{l}\text { No. of } \\
\text { pulses }\end{array}$ & $\begin{array}{l}\text { Current } \\
(\mathrm{kA})\end{array}$ & $\begin{array}{l}\text { Weld } \\
\text { time }(\mathrm{ms})\end{array}$ & $\begin{array}{l}\text { Cool time } \\
(\mathrm{ms})\end{array}$ & $\begin{array}{l}\text { Force } \\
(\mathrm{kN})\end{array}$ & $\begin{array}{l}\text { Hold time } \\
(\mathrm{ms})\end{array}$ \\
\hline 2 & 9.5 & 200 & 33 & 5.5 & 167 \\
\hline
\end{tabular}

Table 1 Chemical composition (wt\%) of examined steel

\section{SN Applied Sciences}

\begin{tabular}{lllllll}
\hline $\mathrm{C}$ & $\mathrm{Mn}$ & $\mathrm{Si}$ & $\mathrm{Al}$ & $\mathrm{Cr}$ & $\mathrm{Mo}$ & $\mathrm{Fe}$ \\
\hline 0.20 & 2.2 & 1.6 & - & - & - & Bal. \\
\hline
\end{tabular}


After cross-sectioning, all samples were hot mounted, ground, polished to a $1 \mu \mathrm{m}$ diamond finish using standard metallographic techniques. After polishing the crosssections were rinsed with methanol and etched with $5 \%$ nital for $2 \mathrm{~s}$ to reveal the weld nugget. An optical microscope at $200 \times$ optical magnification was used to observe all revealed LME cracks on the cross-section and the crack depths were measured using Image ${ }^{\circledR}$ software. Crack depth was measured as the straight line distance from the crack opening to the crack root as per previous work [6] and shown in Fig. 2. After the cracks in each weld crosssection were measured, cracking severity was calculated using the cracking index $(\mathrm{Cl})$, a measure that combines the depth and number of cracks observed from a particular

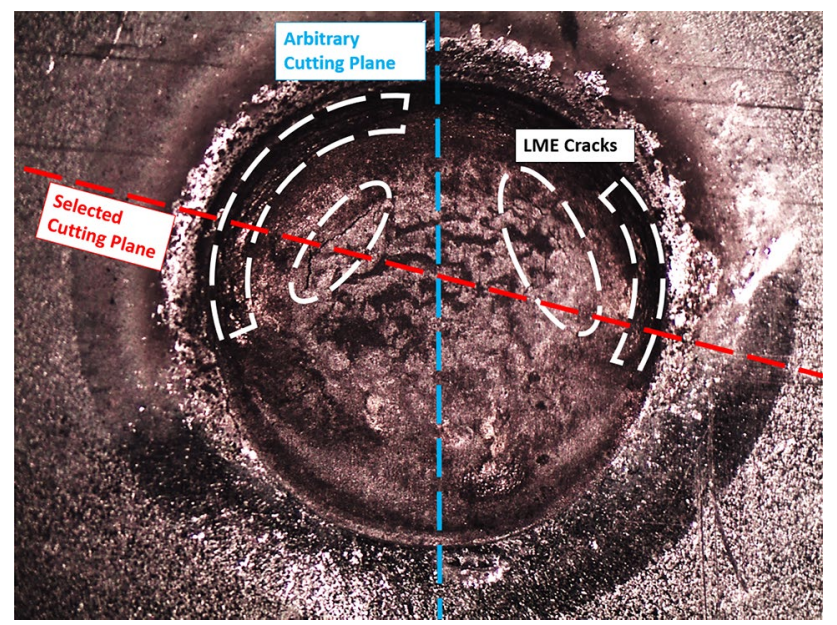

Fig. 1 Cutting planes using the selected cutting plane and arbitrary cutting plane methods
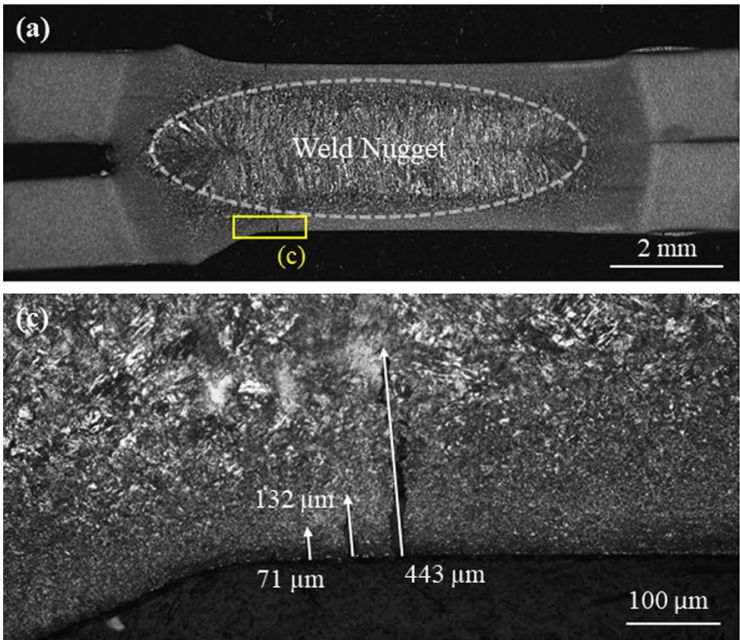

Fig. 2 Examples of cross-sections of cracked welds using the a selected and $\mathbf{b}$ arbitrary cutting plane methods, with increased magnification images of the weld shoulder from cross-sections $c$ weld into one value that has been shown to relate to strength loss in spot welds pulled in tensile shear [6]. The cracking index is defined in Eq. (1)

$C I=\frac{n L}{\tau}$

where $n$ is the mean number of measured cracks per sample, $L$ is the lognormal median (geometric mean) crack depth, and $\tau$ is the sheet thickness. A higher crack index indicates more severe LME cracking. Standard statistical methods were used to test the data. The specific tests are noted in the Results and Discussion section. The standard deviation associated with the lognormal median crack length was calculated using the bootstrap method with 250 datasets of the same number of values as the original measurements; 61 and 24 for the cases of the selected and arbitrary cutting planes, respectively. All error bars represent a $95 \%$ confidence interval and all confidence tests were calculated using a $5 \%$ significance level.

\section{Results and discussion}

\subsection{Distribution of cracks and LME severity measurements}

The distribution of cracks measured from the cross-section of both welds were compared and it was immediately seen that the selected cutting plane revealed more cracks, and the observed cracks were deeper than seen on the arbitrary cutting plane (see Fig. 3), which agrees with the qualitative observations of the cross-sections in Fig. 2. The crack distribution from the selected cutting plane also showed
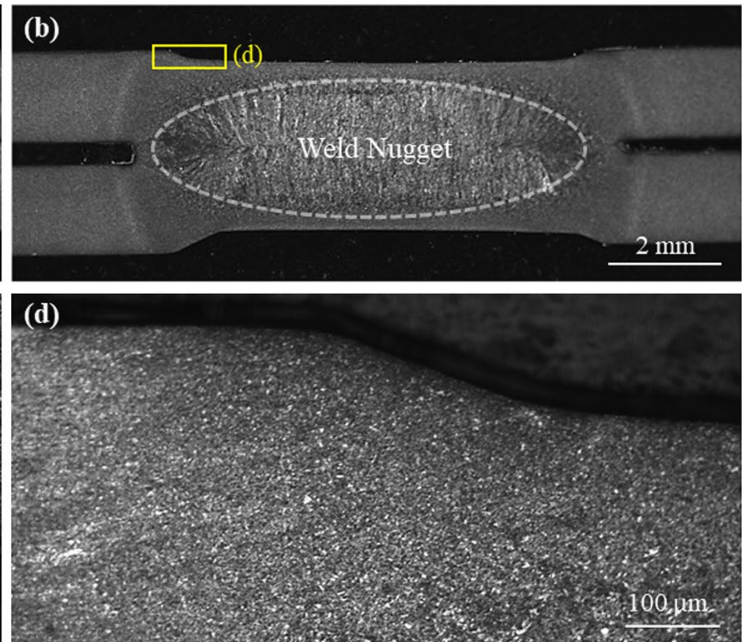

using the selected and $\mathbf{d}$ arbitrary cutting planes. Examples of how crack depth was measured may be seen on inset (c) 


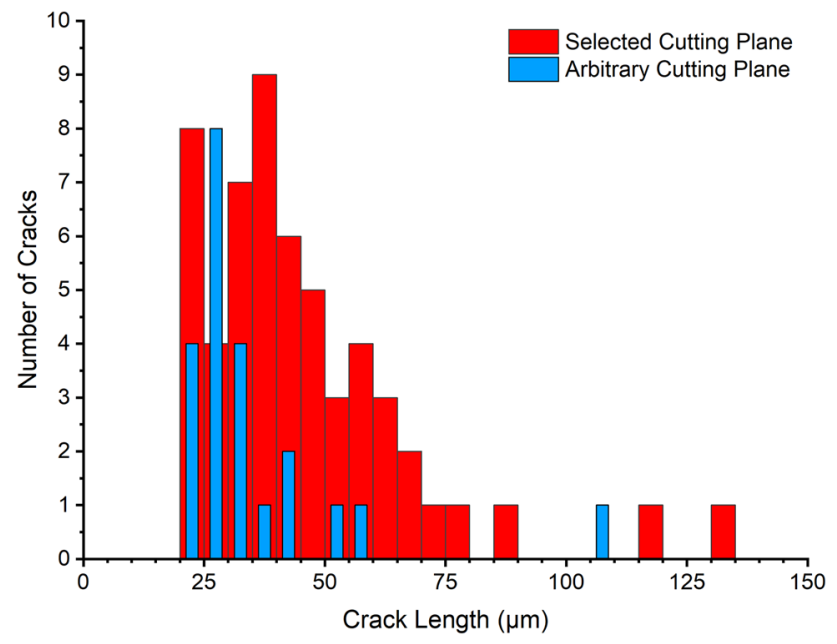

Fig. 3 Histogram of crack distribution measured from cross-sections chosen using both the selected and arbitrary cross-section planes a higher number of small cracks $<25 \mu \mathrm{m}$ as well as much larger cracks. Cross-sections from the selected cutting planes contained 5 cracks that were deeper than $150 \mu \mathrm{m}$ (truncated from Fig. 3), whereas only 1 crack deeper than $150 \mu \mathrm{m}$ was seen from observations made on the arbitrary cutting plane.

To determine the cracking severity, the lognormal median crack depth and cracks per welds were calculated from both sets of cross-sections. Similar to the crack distribution (Fig. 3), the cracks measured from the selected cutting plane were both deeper on average and more numerous than cracks observed from the arbitrary cutting plane (see Fig. 4). The cracks observed from the selected cutting planes had an average crack depth of $49.5 \mu \mathrm{m}$ and the welds contained an average of 10.2 cracks per weld ( 61 cracks total), whereas the arbitrary cutting planes showed 4.0 cracks per weld ( 24 cracks total), which had an average depth of $39.7 \mu \mathrm{m}$. When compared using a T-test, it was seen that both differences in measured crack depth and crack frequency measured using the two methods were significantly different. It should also be noted that the

Table 3 Uncertainty associated with LME cracking metrics from welds prepared using the selected and arbitrary cutting plane methods

\begin{tabular}{|c|c|c|c|c|c|c|c|}
\hline \multirow[t]{2}{*}{ Cross-sectioning methodology } & \multirow[t]{2}{*}{$\begin{array}{l}\text { Degrees of } \\
\text { freedom }\end{array}$} & \multicolumn{2}{|c|}{$\begin{array}{l}\text { Log normal median crack } \\
\text { depth }(\mu \mathrm{m})\end{array}$} & \multicolumn{2}{|c|}{ Cracks per weld } & \multicolumn{2}{|c|}{ Cracking index } \\
\hline & & $\begin{array}{l}\text { Standard } \\
\text { deviation }\end{array}$ & $\begin{array}{l}95 \% \text { confi- } \\
\text { dence interval }\end{array}$ & $\begin{array}{l}\text { Standard } \\
\text { deviation }\end{array}$ & $\begin{array}{l}95 \% \text { confi- } \\
\text { dence interval }\end{array}$ & $\begin{array}{l}\text { Standard } \\
\text { deviation }\end{array}$ & $\begin{array}{l}95 \% \\
\text { confidence } \\
\text { interval }\end{array}$ \\
\hline Selected cutting plane & 60 & 4.0 & 1.0 & 2.8 & 2.2 & 0.091 & 0.024 \\
\hline Arbitrary cutting plane & 23 & 6.8 & 2.7 & 2.4 & 2.0 & 0.063 & 0.027 \\
\hline
\end{tabular}
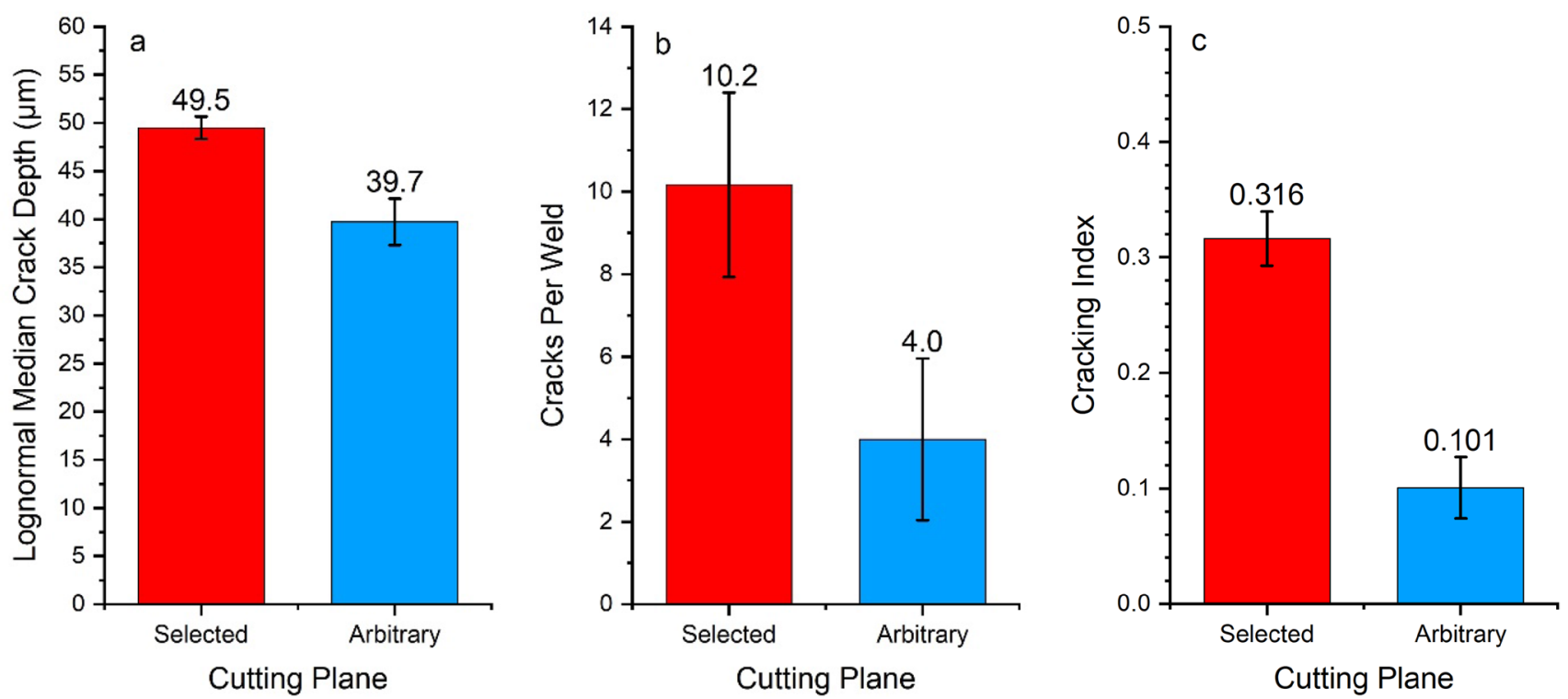

Fig. 4 Comparison of the a lognormal median of measured cracks depths, $\mathbf{b}$ cracks per welds and $\mathbf{c}$ cracking index measured from welds cross-sectioned from both selected and arbitrary cross-sectioning planes 
distribution of cracking data (standard deviation) of the crack depth measurements calculated from the selected cutting plane was significantly narrower than that calculated from the cracks measured from the arbitrary cutting planes (see Table 3), as determined by the F-Test (significance value of 0.002 ). This had the effect of increasing the confidence interval size for the average depth of cracks measured from the arbitrary cutting plane, decreasing the certainty of average (wider error bars).

The cracking index for the welds were calculated from the crack depth and frequency data from each of the crosssections using Eq. (1). As would be expected, the selected cutting plane had a much higher cracking index (0.32) than the arbitrary cutting plane (0.10), as may be seen in Fig. 4c. This was due to the arbitrary cutting plane having fewer and shallower cracks than found on the selected cutting plane. It should be remembered though that all welds were made using the same welding conditions, so the LME crack distribution throughout each weld, both observed and unobserved, should be equivalent. However, the difference in the measured LME severity index between the two observed planes shows that there is a much higher density of cracks below the large visible surface cracks than may be found on arbitrary cross-section planes. This is logical as LME cracking initiates from the material surface when $\mathrm{Zn}$ from the coating degrades the surface grain boundaries [30]. Therefore, cracks must be open to the surface. It stands to reason that neither cross-sectioning technique provides a true picture of the cracking distribution within the weld, but it is also logical that the selected cutting plane represents a worst-case scenario of the local crack distribution.

Although the $\mathrm{Cl}$ combines crack depth and crack frequency in single measurement [Eq. (1)], it is interesting to note that even with higher uncertainty (confidence interval) associated with the crack depths measured from the arbitrary cutting plane than the selected cutting plane, there was not a significant difference between the uncertainty of the $\mathrm{Cl}$ results associated with the two cutting planes. This is most likely due the large uncertainty associated with the crack frequency measurements (Fig. 4b) and the fact that when multiplying numbers with associated uncertainties, part of the calculation to determine the resultant uncertainty is multiplying the variance by the multiplied quantity (lognormal median depth and cracks per weld in this case). As the multiplied quantities for the arbitrary cutting plane were small compared to those of the selected cutting plane, the resultant uncertainty was also small. However, even though the uncertainties associated with the two planes are similar, it should be noted that the due to the lower $\mathrm{Cl}$ value resulting from the arbitrary cutting plane, the uncertainty is a much larger fraction of the total cracking severity measurement. In the case of the data from the arbitrary plane, the confidence interval represents $27 \%$ of the $\mathrm{Cl}$ measurement, whereas it is only $8 \%$ in the case of the $\mathrm{Cl}$ measurements from selected plane. This large relative uncertainty associated with the arbitrary cross-section crack data will have large implications on the ability to make decisions from this data.

\subsection{Influence of cutting plane on improving measurement precision}

As LME cracking has been shown to affect tensile shear strength $[5,8,9]$ and the drop in strength may be predicted from LME cracking data [6], it is important to have precise knowledge of the LME cracking conditions within a weld. To understand the how the $\mathrm{Cl}$ data calculated from the measurements taken from the selected and arbitrary cutting planes impacts interpretation of post-weld properties, the data presented in Fig. 4c was compared to the correlation Wintjes et al. [6] made between weld strength loss and $\mathrm{Cl}$ (Fig. 5). The data from the present study was plotted on Fig. 5 under the assumption that the selected plane data would have a strength loss predicted by Wintjes et al. [6], as both studies used similar welding methodologies and the cross-sectioning technique used by Wintjes et al. was similar to the selected cross-section method, and the arbitrary cutting plane data should exhibit the same strength loss exhibited by the selected cutting plane data, as all welds were made using the same welding parameters. The strength uncertainty was calculated as the predicted strength values associated with the cracking index values at the extreme ends of the $95 \%$ confidence interval of both data points, as shown by the dotted box around the points associated with the present study. By plotting these two points, it may be seen that both methodologies should follow separate strength-loss/crack index correlations, assuming the linear trend seen for the selected cutting plane is also true for arbitrary cutting planes. However, due to the higher relative uncertainty of the $\mathrm{Cl}$ measured from the arbitrary planes there is also less certainty about the strength loss exhibited by the joint. The predictions of percentage of post-weld strength loss when compared to an uncracked weld made from the $\mathrm{Cl}$ value calculated using the selected cutting plane data is accurate to $\pm 0.8 \%$, whereas the $\mathrm{Cl}$ value calculated from the arbitrary cutting planes can only predict strength loss within $\pm 2.8 \%$. The total range of the uncertainty in the case of the arbitrary selected cutting planes represents almost $50 \%$ of the predicted strength loss value. The strength loss uncertainty is shown by the vertical arrows in the Fig. 5; an inset is added to clarify the uncertainty associated with measurements from the selected cutting planes. As indicated earlier, the selected plane method of LME crack analysis may provide 
Fig. 5 Correlation between cracking index and percentage tensile shear strength loss for various steels with data included from the present study, with an inset showing a higher resolution view of points from cracking index values from 0.25 to 0.40 . Adapted from [6]

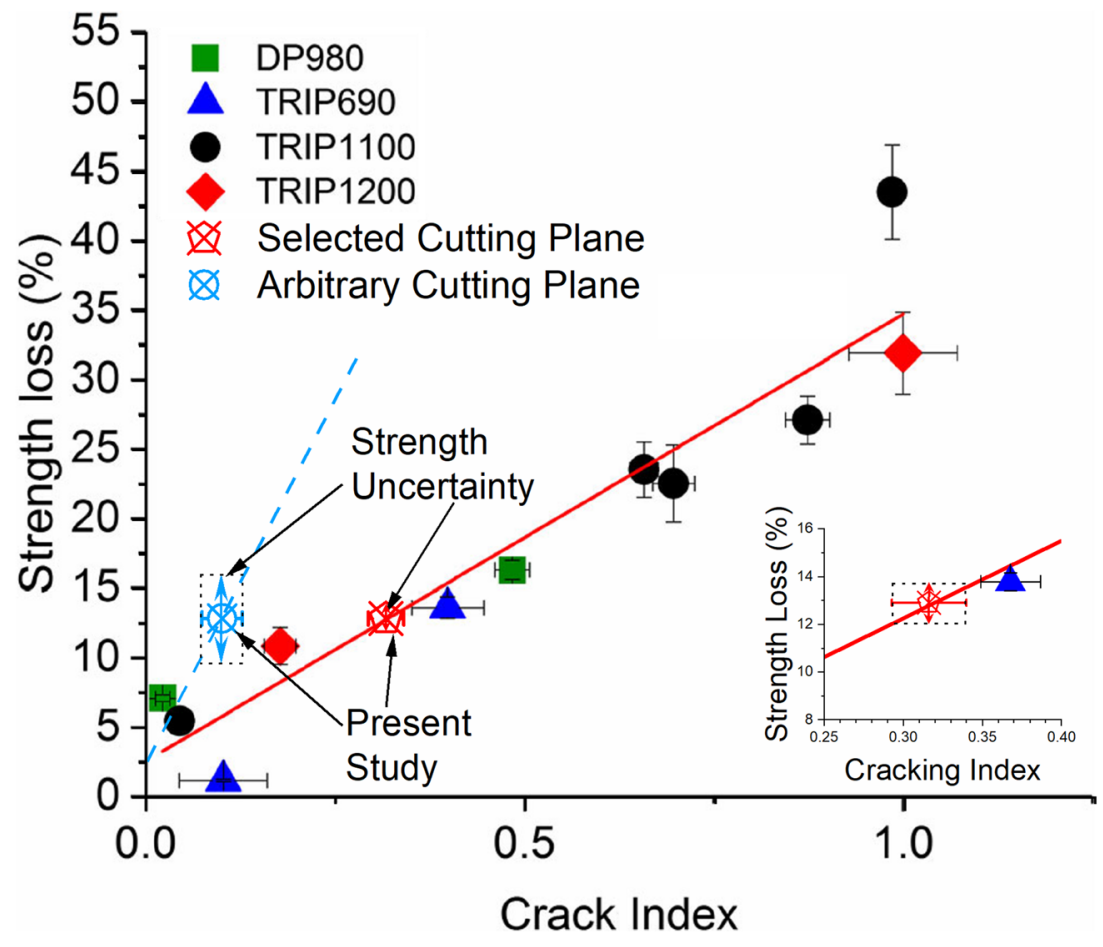

a worst-possible case of local cracking distribution, however, this example shows how exaggerating the measured cracking severity helps to improve measurement resolution. In this case, the exaggerated cracking metric improved mechanical property predictions, but it may also be used to improve resolution when understanding how welding parameter changes affects LME crack severity.

The use the selected cutting plane to measure the most severe local cracking in the weld will also decrease the necessary number of welds to be examined to ensure accurate results. The level of certainty of the predicted cracking severity $(\mathrm{Cl})$ depends on two things: the standard deviation of the measurements, and the number of cracks measured. Although the standard deviation of the $\mathrm{Cl}$ calculation using the selected cutting plane method is higher than that for the $\mathrm{Cl}$ using the arbitrary plane method, 0.091 and 0.063 , respectively (see Table 3 ), the selective cutting plane method revealed about 2.5 times the number of cracks compared to the arbitrary cutting plane method. This means that the averages used to calculate the $\mathrm{Cl}$ are based on a greater number of measured cracks, so the associated uncertainty decreases. From cracks in the present study it may be seen that using the selected cutting plane method drastically reduces the number of cross-sections needed to precisely predict cracking severity. Using the welds in the present study as an example, when the selected cutting plane method is used to reveal LME cracks, only 4 welds are needed to calculate $\mathrm{Cl}$ with a confidence interval of $\pm 10 \%$, whereas using the arbitrary cutting plane method 38 welds are needed (see Fig. 6a). If strength degradation is being calculated, then prediction becomes even more accurate as the slope of the strengthloss $\mathrm{Cl}$ curve in Fig. 5 is shallow for LME cracks measured using the selected cross-section plane. In this case, analysing 4 welds cross-sectioned using the selected cutting plane will enable of a strength loss prediction of $\pm 1 \%$, whereas 40 welds are needed to calculate strength loss to the same precision using the arbitrary cutting method (see Fig. 6b).

\section{Conclusions}

With the large amount of work being done to understand and reduce LME in the automotive sector, there have been many methodologies used to characterize cracking severity. Due to the need to understand crack depth and carry-out cracking analysis in a time-efficient manner, the majority of LME crack characterization is carried out from weld cross-sections. However, this requires researchers to choose where to cross-section the weld. The current study compared the common methods used to choose the cross-sectioning plane for crack analysis: the selected method, chosen to intersect the largest crack, and the arbitrary method, chosen at random without regard to the location of surface cracks. It was found that analyzing LME cracks using the selected cutting plane was far superior to the arbitrary cutting plane. The selected cutting plane revealed more and deeper cracks than were found on the arbitrary cutting plane. This 

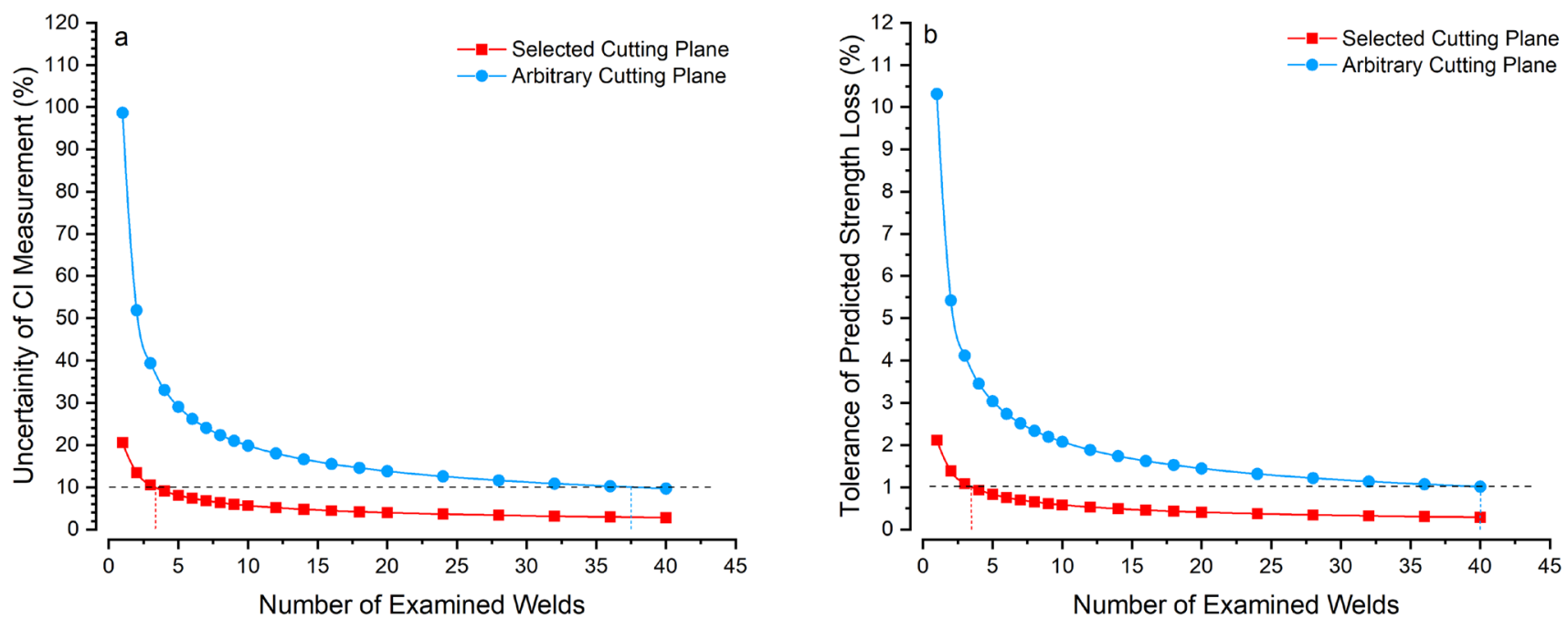

Fig. 6 Predicted uncertainty associated with a cracking index calculation and $\mathbf{b}$ tensile shear strength loss associated with LME cracking from the number of welds examined to determine weld cracking severity

resulted in a higher cracking severity measurement with a lower relative uncertainty. The lower relative uncertainty enabled higher measurement precision, needing fewer samples to examine to achieve acceptable results. Furthermore, this study showed that crack distribution in the weld is not uniform. Although some work may seek to understand the global cracking distribution in the weld, it is thought that the worst-case-scenario measured using the selected cutting plane not only results in more conservative results when predicting the effect of LME cracking on post-weld performance, but it also allows a higher measurement resolution, requiring fewer samples to be analyzed to understand how crack reduction methodologies affect LME cracking severity. Adoption of the selected cutting plane to analyze LME cracking will improve the resolution of LME crack measurements. This will better show how improved materials and weld procedures that are being tested with the objective of reducing LME are effective, which will reduce the time needed to address this issue. This will, in-turn, speed the adoption of high strength AHSS into automotive designs, leading to the associated decreases in vehicle emissions and increases in passenger safety.

Acknowledgements The authors would like to thank ArcelorMittal Dofasco, the International Zinc Association and the Natural Sciences and Engineering Research Council of Canada (NSERC) for funding this work.

Funding This work was funded through awards from ArcelorMittal Dofasco, the International Zinc Association and a Collaborative Research and Development Grant provided by the Natural Sciences and Engineering Research Council of Canada (NSERC) (Grant No. CRDPJ 552526-17).

\section{Declarations}

Conflict of interest The author declare that they have no conflict of interest.

Open Access This article is licensed under a Creative Commons Attribution 4.0 International License, which permits use, sharing, adaptation, distribution and reproduction in any medium or format, as long as you give appropriate credit to the original author(s) and the source, provide a link to the Creative Commons licence, and indicate if changes were made. The images or other third party material in this article are included in the article's Creative Commons licence, unless indicated otherwise in a credit line to the material. If material is not included in the article's Creative Commons licence and your intended use is not permitted by statutory regulation or exceeds the permitted use, you will need to obtain permission directly from the copyright holder. To view a copy of this licence, visit http://creativecommons. org/licenses/by/4.0/.

\section{References}

1. Kim YG, Kim IJ, Kim JS et al (2014) Evaluation of surface crack in resistance spot welds of zn-coated steel. Mater Trans 55:171175. https://doi.org/10.2320/matertrans.M2013244

2. Choi DY, Sharma A, Uhm SH, Jung JP (2019) Liquid metal embrittlement of resistance spot welded 1180 TRIP steel: effect of electrode force on cracking behavior. Met Mater Int 25:219-228. https://doi.org/10.1007/s12540-018-0180-x

3. Bhattacharya $D$ (2018) Liquid metal embrittlement during resistance spot welding of $\mathrm{Zn}$-coated high-strength steels. Mater Sci Technol 34:1809-1829

4. He L, DiGiovanni C, Han X et al (2019) Suppression of liquid metal embrittlement in resistance spot welding of TRIP steel. Sci Technol Weld Join 24:579-586. https://doi.org/10.1080/13621 718.2019.1573011 
5. DiGiovanni C, Biro E, Zhou NY (2019) Impact of liquid metal embrittlement cracks on resistance spot weld static strength. Sci Technol Weld Join 24:218-224

6. Wintjes E, DiGiovanni C, He L et al (2019) Quantifying the link between crack distribution and resistance spot weld strength reduction in liquid metal embrittlement susceptible steels. Weld World 63:807-814. https://doi.org/10.1007/s40194-019-00712-5

7. American Welding Society (2013) D8.1M:2013 specification for automotive weld quality resistance spot welding of steel. American Welding Society, Miami

8. Choi D, Uhm S, Enloe C et al (2018) Liquid metal embrittlement of resistance spot welded 1180TRIP steel-effects of crack geometry on weld mechanical performance. In: Contributed papers from materials science and technology 2017 (MS\&T17). MS\&T17, Pittsburg, pp 454-462

9. DiGiovanni C, Han X, Powell A et al (2019) Experimental and numerical analysis of liquid metal embrittlement crack location. J Mater Eng Perform 28:2045-2052. https://doi.org/10.1007/ s11665-019-04005-2

10. Benlatreche $Y$, Ghassemi-Armaki H, Duchet M et al (2017) Spotweld integrity of $\mathrm{Zn}$-coated 3rd gen. advanced high strength steels in presence of LME. In: International automotive body congress, IABC 2017

11. Murugan SP, Mahmud K, Ji C et al (2019) Critical design parameters of the electrode for liquid metal embrittlement cracking in resistance spot welding. Weld World 63:1613-1632. https:// doi.org/10.1007/s40194-019-00797-y

12. Vijayan V, Murugan SP, Son SG, Do PY (2019) Shrinkage void formation in resistance spot welds: its effect on advanced high-strength-steel weld strength and failure modes. J Mater Eng Perform 28:7514-7526. https://doi.org/10.1007/ s11665-019-04465-6

13. Frei J, Biegler $M$, Rethmeier $M$ et al (2019) Investigation of liquid metal embrittlement of dual phase steel joints by electro-thermomechanical spot-welding simulation. Sci Technol Weld Join 24:624-633. https://doi.org/10.1080/13621718.2019.1582203

14. Chaudhuri S, Crump J, Reed PAS, Mellor BG (2019) High-resolution 3D weld toe stress analysis and ACPD method for weld toe fatigue crack initiation. Weld World 63:1787-1800. https://doi. org/10.1007/s40194-019-00792-3

15. Wintjes E, DiGiovanni C, He L et al (2019) Effect of multiple pulse resistance spot welding schedules on liquid metal embrittlement severity. J Manuf Sci Eng 141:101001. https://doi.org/10. $1115 / 1.4044099$

16. Lee $\mathrm{H}$, Jo MC, Sohn SS et al (2019) Microstructural evolution of liquid metal embrittlement in resistance-spot-welded galvanized twinning-induced plasticity (TWIP) steel sheets. Mater Charact 147:233-241. https://doi.org/10.1016/j.matchar.2018. 11.008

17. Choi D-Y, Uhm S-H, Enloe CM, Lee H, Kim G, Horvath C (2017) Liquid Metal Embrittlement of Resistance Spot Welded 1180TRIP Steel- Effects of Crack Geometry on Weld Mechanical Performance. In: Materials Science and Technology, pp 454-462

18. Gaul H, Weber G, Rethmeier M (2011) Influence of HAZ cracks on fatigue resistance of resistance spot welded joints made of advanced high strength steels. Sci Technol Weld Join 16:440445. https://doi.org/10.1179/1362171810Y.0000000031

19. Ling $Z$, Chen $T$, Kong $L$ et al (2019) Liquid metal embrittlement cracking during resistance spot welding of galvanized Q\&P980 steel. Metall Mater Trans A Phys Metall Mater Sci 50:5128-5142. https://doi.org/10.1007/s11661-019-05388-6

20. Taram A, Roquelet C, Meilland P et al (2018) Nondestructive testing of resistance spot welds using eddy current thermography. Appl Opt 57:D63. https://doi.org/10.1364/ao.57.000d63

21. Wintjes E, DiGiovanni C, He L et al (2019) Effect of multiple pulse resistance spot welding schedules on liquid metal embrittlement severity. J Manuf Sci Eng 141:1-9. https://doi.org/10. 1115/1.4044099

22. Benlatreche Y, Dupuy T, Ghassemi-Armaki H et al (2019) Methodology for liquid metal embrittlement (LME) evaluation of coated steels during spot welding. In: 72 nd annual assembly of the international institute of welding, Bratislava

23. Sigler DR, Schroth JG, Yang W et al (2008) Observations of liquid metal-assisted cracking in resistance spot welds of zinc-coated advanced high-strength steels. In: Sheet metal welding conference XIII. Livonia, pp 1-1

24. Tumuluru M (2019) Effect of silicon and retained austenite on the liquid metal embrittlement cracking behavior of GEN3 and high-strength automotive steels. Weld J 98:351S-364S. https:// doi.org/10.29391/2019.98.029

25. DiGiovanni C, Bag S, Mehling C et al (2019) Reduction in liquid metal embrittlement cracking using weld current ramping. Weld World 63:1583-1591. https://doi.org/10.1007/ s40194-019-00790-5

26. Ashiri R, Shamanian M, Salimijazi HR et al (2016) Liquid metal embrittlement-free welds of $\mathrm{Zn}$-coated twinning induced plasticity steels. Scr Mater 114:41-47

27. He L, DiGiovanni C, Han X et al (2019) Suppression of liquid metal embrittlement in resistance spot welding of TRIP steel. Sci Technol Weld Join. https://doi.org/10.1080/13621718.2019. 1573011

28. Tolf E, Hedegård J, Melander A (2012) Surface breaking cracks in resistance spot welds of dual phase steels with electrogalvanised and hot dip zinc coating. Sci Technol Weld Join 18:25-31. https://doi.org/10.1179/1362171812Y.0000000068

29. American Welding Society (2012) AWS D8.9:2012 Test Methods for Evaluating the Resistance Spot Welding Behavior of Automotive Sheet Steel Materials. American Welding Society, Miami, FL

30. Beal C, Kleber X, Fabregue D, Bouzekri M (2012) Embrittlement of a zinc coated high manganese TWIP steel. Mater Sci Eng A 543:76-83. https://doi.org/10.1016/j.msea.2012.02.049

Publisher's Note Springer Nature remains neutral with regard to jurisdictional claims in published maps and institutional affiliations. 